\title{
FIIISA
}

\section{Rational Agents, Contract Curves, and Inefficient Compromises}

Gregory E. Kersten and Sunil J. Noronha

RR-99-8

August 1999

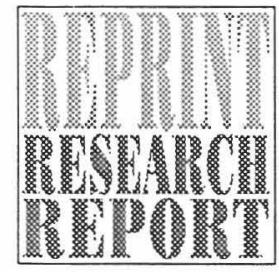




\title{
RATIONAL AGENTS, CONTRACT CURVES, AND INEFFICIENT COMPROMISES
}

\author{
Gregory E. Kersten \\ School of Business, Carleton University, Ottawa, Canada and \\ International Institute for Applied Systems Analysis \\ Laxenburg, Austria \\ Sunil J. Noronha \\ IBM T.J. Watson Research Center, New York, USA
}

RR-99-8

August 1999

Reprinted from IEEE Transactions on Systems, Man, and Cybernetics - Part A: Systems and Humans, Volume 28, Number 3, pp. 326-338, May 1998.

International Institute for Applied Systems Analysis, Laxenburg, Austria Tel: +432236 807 Fax: +43223673148 E-mail: publications@iiasa.ac.at 
Research Reports, which record research conducted at IIASA, are independently reviewed before publication. Views or opinions expressed herein do not necessarily represent those of the Institute, its National Member Organizations, or other organizations supporting the work.

Reprinted with permission from IEEE Transactions on Systems, Man, and Cybernetics Part A: Systems and Humans, Volume 28, Number 3, pp. 326-338, May 1998.

Copyright (C) 1998, IEEE.

All rights reserved. No part of this publication may be reproduced or transmitted in any form or by any means, electronic or mechanical, including photocopy, recording, or any information storage or retrieval system, without permission in writing from the copyright holder. 


\title{
Rational Agents, Contract Curves, and Inefficient Compromises
}

\author{
Gregory E. Kersten and Sunil J. Noronha, Member, IEEE
}

\begin{abstract}
Several studies of two-party negotiations have shown that negotiators more often than not reach inefficient compromises. We analyze the circumstances under which rational agents make inefficient compromises and refrain from improving them. We do this by describing and interpreting various negotiation situations and by developing formal constructs and theorems for determining the character of a negotiation situation. Key among these concepts is the notion of opposition. Although opposition is defined in terms of utility functions, it is more fundamental in the sense that it is more intuitive to decision makers and can be used in contexts in which the parties' utilities are unknown or are partially known. The effects of various rationality assumptions on efficiency and their implications for negotiation support systems are discussed. We argue that the prescriptive/descriptive approach advocated by negotiation analysts lacks sufficient explanatory powers to be effectively used in negotiation support and that negotiation support systems should not constrain the parties to the set of efficient points.
\end{abstract}

Index Terms-Bargaining, conflict analysis, decision and negotiation support, efficiency, negotiation analysis, negotiations, opposition, rationality, utility.

\section{INTRODUCTION}

A LEMI, Fos, and Lacorte [1] conducted experiments with six professionals, some of whom were professional negotiators or were often involved in negotiations. These experiments showed that the negotiators more often than not reached inefficient compromises. In [1], the authors state that "If physicians and managers can arrive at Pareto-optimal contracts, then their negotiations are effective." Similar results have been reported in [2] with only $10 \%$ of 90 dyads achieving an efficient compromise [3]-[5].

Teich et al. [6] conducted experiments with students using two negotiation support systems. They also often did not achieve an efficient (i.e., Pareto-optimal) compromise. Moreover, when the students were presented with an efficient compromise they did not want to move from the inefficient to the efficient compromise.

Manuscript received January 19, 1996; revised July 5, 1997. This work was supported by strategic grants from the Natural Sciences and Engineering Research Council of Canada and the Social Sciences and Humanities Council of Canada. An earlier version of this paper was presented at the MCDM Conference, Coimbra, Ponugal, 1994.

G. E. Kersten is with the School of Business, Carleton University, Ottawa, Ont., KIS 5B6, Canada. He is also with the DAS Project, International Institute for Applied Systems Sciences, Laxenburg, Austria.

S. J. Noronha was with the School of Business, Carleton University, Ottawa, Ont., Canada. He is now with the IBM T. J. Watson Research Center, Yorktown Heights, NY 10532-1507 USA

Publisher Item Identifier S 1083-4427(98)02745-3.
In a discussion on rational choice in games McClennen [7, p. 259], says the following:

"When one turns to consider nonstrictly competitive games, the assumption that rational players are bound by the principle of maximization of expected utility proves to be quite paradoxical. Except under very special circumstances, it ensures that rational interaction will fail to satisfy the criterion of Pareto optimality."

This appears to be an unusual remark and one that has no support in the literature on prescriptive decision making, rational agents and the application of decision analysis to negotiations [5], [8]. On the other hand, this remark, as indicated above, is substantiated by real-life negotiation and by laboratory experiments. In this paper, we analyze the circumstances under which rational agents do not choose efficient compromise proposals and achieve an inefficient compromise. We also make the argument that rational agents who have achieved an inefficient compromise may not want to change it and accept an efficient one, and present reasons for such behavior. We take a cognitive perspective and present formulae to model the reasons underlying the agents' behavior. The discussion is illustrated with a simple case of two-issue bilateral negotiations.

The objectives of this paper are two-fold. One is to describe and interpret different negotiation situations and study situations in which rational agents fail to achieve efficient compromises. The other is to argue that the asymmetrically prescriptive/descriptive approach advocated by negotiation analysts [5], [8], [9] lacks sufficient explanatory power and a consistent analytic framework to be effectively used in negotiation support. Negotiation analysis does not take into account the rationalities underlying the opponent's behavior, nor does it allow for multiple or divergent rationalities, which are typical of human decision making [10]-[13]. We outline how models of the opponent's behavior can be constructed from partial information.

We take a prescriptive/normative perspective. The normative perspective is in the assumptions of both parties being rational, using logically consistent decision procedures, and wanting to achieve an efficient compromise. The supported party is being offered prescriptions on how to make better decision leading to an efficient compromise. There are several significant distinctions to the traditional normative approaches, prime among which is that we do not posit a single gametheoretic rationality with a known utility function.

Negotiation analysts using the traditional approach live with the inconsistency of using a model which assumes complete 
knowledge of the decision maker and the problem and therefore presumes to give complete advice, but of having in reality to make considerable practical modifications and adaptations since the problem is never complete. This lack of completeness encompasses not only the opponent but also the supported decision maker. Therefore, Lax and Sebenius [8], [14] amend formal representations with numerous prescriptions that are more in line with the rules and principles proposed by Fisher, Ury and their associates [15], [16] than with the prescriptions of negotiation analysis [5]. Review of the applications and negotiation support systems that are based on negotiation analysis suggests its two main uses: education and research [8], [17], [18].

Our view is that attempts to measure all relevant aspects of the problem are often fruitless, any prescription is only partial and tentative, and that negotiation analysis and support should not constrain the agents to the set of efficient offers. We advocate the use of methods that permit formal analysis of whatever qualitative information is available. In particular, the approach based on the concept of opposition that we propose has the advantage of supporting analysis without requiring precise elicitation of the parties' utility curves over the entire space of feasible compromises. Moreover, it allows one to use many attributes of the problem, process, and decision makers that the parties are unable to incorporate into their respective utilities. Indeed, the concept of opposition or motivational force can be viewed as more fundamental in the sense that it exists for problems for which utility functions cannot exist.

This paper is organized as follows. In Section II we present a negotiation case to introduce the concepts and issues considered later. The assumptions and definitions of the nature of opposition are formulated in Section III. Formal conditions for efficient compromises are obtained in Section IV. It has been observed (see, e.g., [19]), that even small changes in the parties' preferences may change their relationship from strong to weak opposition, and these cases are explored in Section IV. We show, further, that the opposition may also change when the parties consider different alternatives while maintaining their preferences. Behavioral reasons that may cause the negotiating agents to resist accepting an efficient compromise in the situation when an inefficient one was negotiated are given in Section V. Acceptance of inefficient compromises by rational agents has significant implications for negotiation analysis and support and some of these implications are presented in Section VI, which concludes the paper.

\section{Two-Party Negotiations}

\section{A. Trade Union Case-Initial Positions}

Consider a simple case of negotiation between trade union and management over only two issues. ${ }^{1}$ The first issue is salary. The second issue involves a bonus which is tied to the production level; the bonus is being paid only after some number of widgets has been produced. The number of

${ }^{1}$ The case presented here is highly stylized. However, from our discussions with a trade union negotiator, the manner-though not the language-in which the issues are discussed and the reasoning process correspond to those experienced in real negotiations.
TABLE I

INTIAL POSTIONS

\begin{tabular}{l|c|c}
\hline & Union (U0) & Management (M0) \\
\hline Salary level & $\$ 7,000$ & $\$ 3,400$ \\
\hline No. of widgets & 1,200 & 5,100 \\
\hline
\end{tabular}

TABLE II

Five Equivalent Alternatives

\begin{tabular}{l|l|l|l|l|l}
\hline Altcmatives & cl & e2 & e3 & e4 & e5 \\
\hline Salary level & $\$ 5,100$ & S 5,200 & $\$ 5,600$ & $\$ 6,100$ & $\$ 6,600$ \\
\hline No. of widgets & 1,200 & 1,800 & 2,700 & 3,500 & 4,200 \\
\hline
\end{tabular}

widgets and the salary level are the subject of negotiation. Not surprisingly, the union wants the highest possible salary level and the lowest number of widgets. The management's interests are opposing. The two sides may have different preferences with respect to each issue, but each party does not know the preferences of the other party, except qualitatively. Moreover, we assume that there are no other issues known or unknown to the party.

The union announced its opening position and the management replied to it. The two offers are presented in Table I.

\section{B. Concessions and Utility Equivalent Alternatives}

The union decides to make a concession and propose a new offer of $(\$ 5600 ; 2700)$. However, a decision analyst is among its membership and he persuades the negotiating team to use his services. The analyst determines, through a series of pairwise comparisons, ${ }^{2}$ that the union's negotiating team is indifferent among five alternatives including the contemplated alternative $(\$ 5600 ; 2700)$. The five equivalent alternatives are given in Table II.

To get an analytical handle on the problem, the analyst attempts to fit a curve to the $e 1-c 5$ point estimates. He chooses a hyperbola, guided both visually by the relative positions of the points and by the asymptotic properties of the curve, and is able to obtain a meaningful interpretation of the components of the resultant expression, as described below. The analyst obtains the union's utility function as

$$
U_{U}(w, s)=15+0.5\left(s+\frac{3.902}{w-5.596}\right)
$$

where $s$ is the salary in thousands of dollars, and $w$ the number of widgets in thousands. The constant 15 and the multiplication factor of 0.5 have no real significance as is usual for utility functions, since utilities are determined unambiguously only

${ }^{2}$ While we do not wish to underplay the difficulty of eliciting preference information such as this, extending our example to model uncertainties and discussing the traditional lottery/fair-bet/revealed preference issues that beset utility theory would considerably lengthen this paper and would be incidental to its purpose-elucidating the properties of opposition and its implications with respect to rationality and inefficiency in even the simplest deterministic case. 


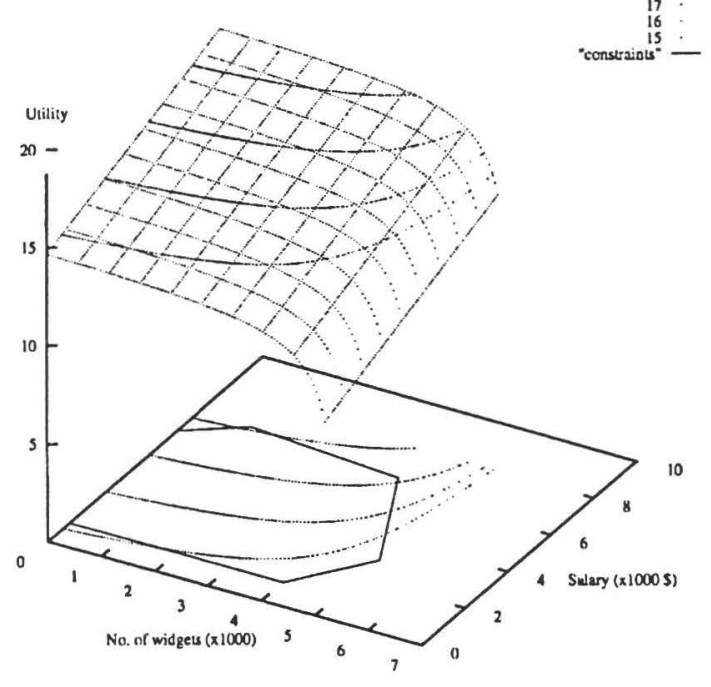

Fig. 1. Union's utility function.

up to a translation and a scaling [20], and have been simply chosen so as to make the three-dimensional (3-D) plot in Fig. 1 easy to view. (For this particular choice of translation and scaling ( 15 and 0.5 , respectively), the fitted curve corresponds to $U_{U}(w, s) \approx 17.1$.) The expression reflects the union's desire for a higher salary, as well as a desire to make as few widgets as possible. The term $(w-5.59)$ in the denominator reflects the fact that it is impossible to increase the number of widgets that can be humanly made beyond a point (about 5000), and causes the utility to fall sharply (or all the level curves to rise steeply near that point).

The union negotiating team appreciates the analyst's work and decides to make an offer $c 2=(1800 ; \$ 5200)$ instead of $e 3=(2700 ; \$ 5600)$ which was earlier contemplated. The analyst, to the surprise of the union team, strongly argues against this offer. He says that selection of this offer may result in a "nonoptimal" (or, strictly speaking, inefficient) compromise. The analyst who during the earlier session with the team had determined the team's utility now wants to present an argument for selecting $c 4=(3500 ; \$ 6100)$. He begins the presentation with Fig. 2 .

The analyst does not know the management's utility function and, therefore, he cannot precisely determine the efficient solutions. However, he knows that the management is interested in both low salary level and high number of widgets before the bonus is paid. Thus, the management's tradeoffs or indifference curves must have a positive slope (i.e., the tangents at various points along the curve must form an acute angle with the horizontal $w$ axis).

Although the analyst does not know the precise tradeoffs, he can hypothesize the extreme values of the tradeoffs, and make conservative estimates of a range of slopes that management's indifference curves must lie within, at any point in the feasible set. Fig. 3 illustrates the various possibilities, including the

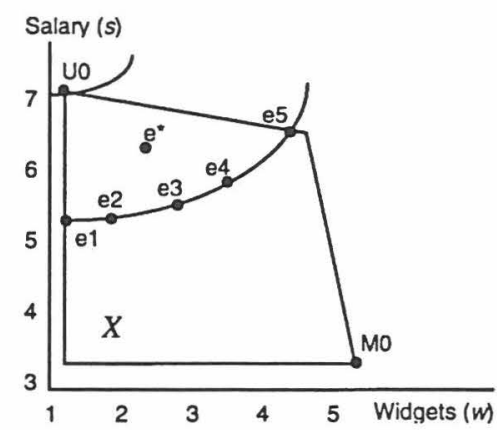

Fig. 2. Mapping of union's utility in offer space.

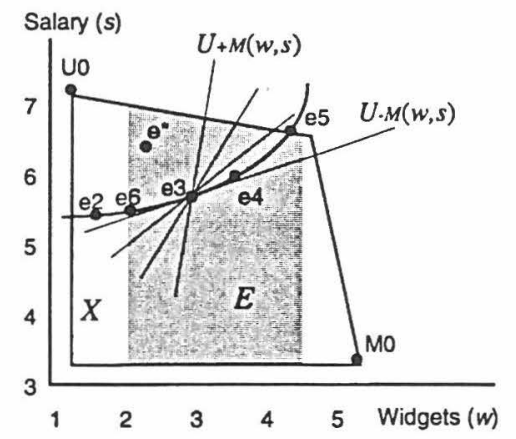

Fig. 3. The range of management's possible indifference curves at $e 3$.

mapping of two extremal utility functions $U_{M}^{+}(s, w)$ and $U_{M}^{-}(s, w)$ that he considers at $e 3$.

Function $U_{M}^{+}(s, w)$ corresponds to the assumption that the management is predominantly interested in widget production and much less in the salary level; while $U_{M}^{-}(s, w)$ reflects the assumption that the management's preference for low salary is much stronger than widgets production. The analyst does not know the form of either of these functions, but merely knows that their gradients (slopes, representing tradeoffs or relative weighting between salary and widgets) must almost certainly be within extreme values $[0.3,3]$ as illustrated. Knowing just this, and the theorem given in Section IV, he can determine the region within which the contract curve $(C C)$ must lie. We call this the region of possibly efficient compromises and it is the shaded area $E$ in Fig. 3. Its computation is described in Appendix A. It is the analyst's contention that since the estimates were conservative, the solutions just within the boundary of $E$ (e.g., e6), while possibly efficient, are less probably efficient than solutions in the interior of $E$, as the former reflect extreme values of management's preferences.

The analyst presents Fig. 3 to the negotiating team and shows that $e 2$ does not lie on any possible $C C$. It means, he explains, that $e 2$ is an inefficient compromise for any possible utility function of the management. The closest and extremal possibly-efficient point which is utility-equivalent to $e 1, e 2, \cdots, e 5$ is $e 6$. He, therefore, is convinced that selection of $e 2$ may "push" the negotiation toward an inefficient compromise. 


\section{First Compromise Proposal}

The union's senior negotiator questions the analyst's reasoning and points out that while the union is indifferent among the five alternatives, the management need not be. The analyst interjects, saying that this is exactly the case and that if the union were to select say, $e 1$, the management may consider such an offer as negotiation in bad faith. This is because, although, $e 1$ brings forth concessions made by the union, it may be considered by the management as a worse offer than the opening position $U 0$. The management's utility level may be higher at $U 0$ than at $e 1$.

The senior negotiator fully agrees with the analyst and says that this is his very reason for selecting $c 2$ and not $c 3, e 4$, or $e 5$. Proposing $e 2$ is constructive because it shows the union's willingness to make concessions, $e 2$ is better for the management than $U O$. At the same time, it gives the union flexibility in its future offers. The union, during the later stages of negotiation, may present compromise proposals which are better for the management but which do not require the union to make any concessions.

The union, for example, can reply with $e 3$ or $e 4$ to the management's counter offer to $e 2$. The selection of one of these alternatives ( $e 3$ or $e 4$ ) would depend on the management's reply to $e 2$; on the degree of their concessions. Moreover, he continues, selection of $e 2$ may lead the management to assume that the union is much less interested in salary level than in the bonus. This may lead the management to put pressure on the union to accept a higher number of widgets. The union may accept it under condition that the management accepts a higher level of salary. This may give the union a strategic advantage over the management.

The analyst listens to the senior negotiator's arguments and suggests that the current utility does not reflect all the preferences of the union. He proposes to include offer flexibility and the probability of management's negative counter-offers to any given offer. This expansion of the utility function is rejected by the union because of its complexity, data requirements and the difficulty of making a meaningful comparison of offer attributes. The senior negotiator states that an attempt to measure everything is impractical and introduces endless decomposition of attributes and the vicious circle of strategic interaction [20].

The analyst then suggests decreasing the concession level earlier agreed to by the union and selecting an alternative which is on or near the efficient frontier. For example, the union may select $c^{*}$, depicted in Fig. 2, as its reply to $M 0$. The union's utility level at $e^{*}$ is higher than at $e 2$, that is $U_{U}\left(e^{*}\right)>U_{U}(e 2)$, and thus-from the union's viewpoint-the concession level is smaller.

The senior negotiator replies that this may significantly weaken the union's arguments in the future. The union, with the selection of $c 2$, makes a significant concession and this, if needed, can be explained to the management. The significant distance from $U O$ to $e 2$ can be easily shown, for example with the use of Fig. 2 with removal of the union's indifference curve. The union, proposing $c 2$, makes concessions measured by the changes in the levels of both negotiating issues (salary and production). These issues are what the two sides are negotiating about. Therefore, a significant change cannot be dismissed by the opponent.

Thus, the senior negotiator argues, choosing $e^{*}$ would amount to making small steps without attempting to build an understanding between the parties, reducing the tensions and making cooperative moves [8]. Offer $e 2$ shows a significant concession made with respect to one issue and a small one to another. It shows the union's willingness to negotiate efficiently but cautiously.

Assume, he continues, that the management replies to $e^{*}$ with an offer which is only marginally better than $M 0$. We would have to reciprocate in kind and the negotiations may take a long time or end in a deadlock. We have our constituency and this negotiation is only a part of our responsibilities. He finishes with questions to the analyst: Can you assure us that the result of "small steps" negotiation will yield a better compromise for the union? Can you assess the increase in the union's utility of a compromise achieved after selecting $e^{*}$ as opposed to the selection of $e^{2}$ ?

Decision analysis does not address this questions and the analyst decides to discontinue the discussion and to concentrate instead on "post-settlement settlement" [5]. He thinks that although the compromise the two parties achieve may be inefficient, he may be able to propose an offer that will benefit both sides.

\section{Offer Exchange and Actual Compromise}

The negotiation continues along the lines proposed by the senior negotiator. The union replies to $M 0=(\$ 3200 ; 5100)$ with $U 1=(\$ 5200 ; 1800)$, (note that $U 1=e 2)$. The management responds with a counter-offer $M 1=(\$ 4000 ; 4500)$ which the union considers as an improvement over $M 0$. However, the union believes that the management's offer does not match the union's concession. The senior negotiator points out that with the earlier selection of $U_{1}$ the union has now flexibility in its response. It may propose an offer which requires a very small or no decrease in its utility while maintaining negotiations in good faith. This is because the management should perceive the new proposal as being significantly better than $U 1$. Moreover, he adds, the counteroffer should visibly reduce the distance between the union's and the management's positions (see Fig. 4) and indicate their willingness to get both parties closer to a compromise.

Taking the above into considerations, the union proposes $U 2=(\$ 5200 ; 2700)$ to which the management replies with $M 2=(\$ 4400 ; 4100)$. Both the union and the management realize that the compromise is close and, after a long meeting, they agree on the compromise $U M=(\$ 4700 ; 3300)$. The negotiation process leading to the compromise $U M$ is illustrated in Fig. 4.

The two parties achieve a compromise that seems to satisfy them. We argue that the union's decisions were based on the rational premises; they included internal aspects of the problem (tradeoffs and utility) and external aspects (opponent's needs, reactions, and other stakeholders). The union made moves analyzing its criteria and determined its utility function. Based 


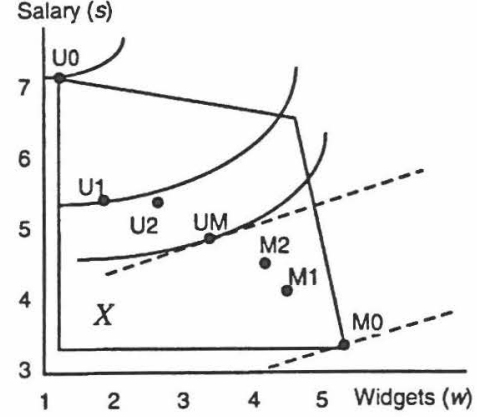

Fig. 4. Union/management negotiations in offer space.

on this analysis, it selected an offer $(U 1)$, with a strategic perspective. This offer, from the decision analysis viewpoint, brought forth concessions that the union was earlier willing to make. The concessions were significant and they could be presented to the management as such. It is also a flexible offer, as it allows the union to make further offers without making any concessions on its part but which would increase the management's utility and thus be considered by the management as concessions. Moreover, at no point in time the union could be accused of making negotiations in bad faith, each of its contemplated or actual offers was getting the two parties closer to a compromise in the sense that it decreased the distance which negotiators may use (e.g., the block measure).

\section{E. Post-Settlement Settlements and Accuracy}

Union and management achieved the compromise $U M$. The analyst now needs to determine if $U M$ is efficient. This is a straightforward exercise that requires the use of Theorem 1 , given in Section IV, if the analytical form of the two utility functions is known. Thus, the analyst must determine the management's utility. Assume, that the management is willing to cooperate and that the analyst determines that the management's indifference curves are as depicted in Fig. 6. This implies that the compromise $U M$ is inefficient. Realizing this he suggests a post-settlement settlement. Offers $D$ or $P 2$ should be considered as a final compromise. He adds that, with the selection of $P 2$, the management will be able to increase its utility value from 25.1 at $U M$ to 26.8 .

The management seems reluctant to accept the analyst's arguments and one of the team members states that he really does not see any improvements. The analyst explains that from his discussion with the team he was able to precisely determine the management's utility and this allowed him to propose a compromise better than $U M$. The member raises the issue of accuracy and says that the tradeoffs really reflect the average perception of all the members and cannot be considered precisely. The member therefore asks the analyst to conduct sensitivity analysis.

The analyst determines the range of changes in the tradeoff values for which $U M$ is efficient and for which the $P 2$ is efficient and presents them to the management team. The discussion then concentrates on the issue whether the trade- off value between widgets and salary is indeed exactly 2.7 . It continues for some time, when the management realizes that this discussion is meaningless because they cannot give a precise value. Then, the analyst asks management to rank the three alternatives $U M, D$, and $P 2$. The management team selects $D$ as a clearly better option than $U M$, but is not able to differentiate between $D$ and $P 2$.

The analyst returns to the union representatives and suggest that, instead of $U M, D$ be selected and that if the union agrees the management may be willing to accept this offer. He points out, that the union prefers $D$ over $U M$ and, therefore, they should accept it, or even suggest $P 2$ as the final compromise. The discussion following the analyst's presentation closely resembles one he earlier had with the management. In effect $U M$ remains the accepted compromise.

Note that we considered here a highly unrealistic situation with the analyst having full information about both parties. Most often the knowledge about the opponent is only partial. As we will show in Section IV, even small changes in the evaluation of the utilities may cause significant changes in the $C C^{\prime} s$ and move the efficient solutions from the boundary to the interior of $X$ or vice versa.

\section{ASSUMPTIONS AND DEFINITIONS}

We consider two party negotiations over issues on which certain constraints are imposed. The negotiations are conducted in the offer space $\Re$ which is an $n$-dimensional space of real numbers. The set $X \subseteq \Re$ of feasible options is defined by constraints which both parties must obey and it is assumed convex. Utilities $U_{M}(x)$ and $U_{U}(x)$, of the two parties are functions defined over $X$ and its "neighborhood;" they are in general convex (or concave) functions. Equality or inequality constraints on these functions therefore define convex curves and regions in $X$.

For the most part, we assume that the parties' utilities are stable over time. This is not really a restriction with respect to our objectives since it is easy to understand changing preferences as a cause of non efficient compromises (the solution to the changed problem is non efficient with respect to the original problem); indeed our intention is to show that even with stable utilities there are reasons for terminating negotiation with an inefficient compromise.

We make the assumption that each party knows his or her own utility function, but not the other party's. This reflects the fact that in real-world negotiations the exact preferences of the other party are incompletely known to each side, and this information is often guarded because it may be disadvantageous to reveal one's true preferences. However, it may be possible to make a qualitative estimate of the other party's utility function, and we will explore this possibility. Indeed, the concepts we develop will make it possible to provide support without having the utility function of either party.

Incomplete information as a cause of inefficiency has been previously studied and is well-known [21]-[24]. These studies demonstrate this in fairly restricted negotiations in which one characteristic is unknown, but strong assumptions are made about the rest of the problem and the negotiators' cognitive 
capabilities. Lax and Sebenius [14, Ch. 7] look at more general negotiations and conclude that under incomplete information, the tension between creating and claiming value is a primary cause of inefficient outcomes.

We are more concerned with the manner in which tactics, strategic moves and cognitive limitations, rather than incomplete information, lead to inefficient compromises. These issues include trust and animosity, external pressure, flexibility considerations, other decision problems and parties encountered, anticipation of future negotiations, and manipulation of the parties' motivations. A typical demonstration will be that a small change in the assessment of the opponent's utility function or additional considerations of the opponent's reactions, can result in a drastic change in the character of the negotiations, e.g., converting it from distributive to cooperative, and turning the compromise from nearly efficient to clearly inefficient.

Definition 1: The $(C C)$ is the subset of the feasible set $X \subseteq \Re$ which contains only efficient solutions.

For unconstrained problems $(X=\Re)$ the $C C$ was first discussed by Edgeworth [25]. In constrained problems $(X \subset$ $\Re$ ), the $C C$ may be contained in $X$, partially contained and partially determined by the frontiers (constraint boundaries) of $X$, or fully located on the frontiers of $X$ [25]. The $C C$ in offer-space corresponds to the Pareto frontier in utility-space. Thus, the Pareto frontier and the $C C$ are fully defined by the two utility functions and $X$.

Further, we characterize the nature of the relationship by defining the opposition between two negotiating parties. We use the term "opposition" in a general sense referring to relationships between the motivations or preferences of the two agents with respect to each other. Intuitively, the strength of opposition is the level of (dis)agreement between the parties at any point $p \in \Re$ or over a region of possible offers, $X \subseteq \Re$. Strict or strong opposition is typical of strictly competitive games when any gain for one party can be achieved only at a loss for another party. At the other extreme, weak or a complete lack of opposition means that both parties simultaneously achieve either losses or gains. This distinction corresponds roughly to the win-lose and winwin types of negotiations. Our formal characterization of opposition allows for a finer distinction of types of negotiation and more comprehensive analysis. In particular, it can help the parties obtain an intuitive, qualitative understanding of how different regions of $X$ are characterized by different levels of opposition, and can thus motivate and guide the search for solutions that release the tension between cooperative versus distributive bargaining.

Since opposition between the two parties can vary considerably over the offer space (with one pair of positions considered highly antagonistic and another pair being highly cooperative), we need to describe the local properties of the relationship. We begin with the opposition at any point of the offer space $\Re$.

Definition 2: The parties are in local strict opposition at a point $x \in \Re$ iff for all points $x^{\prime} \in \Re$ that are sufficiently close to $x$ (i.e., for some $\varepsilon, \varepsilon>0$, such that $\forall x^{\prime}\left\|x^{\prime}-x\right\|<\varepsilon$ ), an increase of one utility can be achieved only at the expense of a decrease of the other utility.
The above definition can be usefully restated using vector calculus as follows. Recall that the level curves or indifference curves of a utility function $U(x)$ are curves of constant utility value $U(x)=c$, for some $c$. The gradient $\nabla U(x)$ at a point $x$ is therefore perpendicular to the level curve of $U(x)$ that goes through $x$. Further, $U(x)$ increases most rapidly at $x$ in the direction of the gradient, and it decreases most rapidly in the opposite direction. The rate of increase in any direction $\boldsymbol{e}$ is given by $\boldsymbol{e} \cdot \nabla U(x)$. As discussed in Section IV, points of local strict opposition (Definition 2) are exactly those at which the gradients of the two utility functions point in opposite directions, i.e., $x \in \Re$ such that $\nabla U_{1}(x)=-k \nabla U_{2}(x), k>0$, and this property could constitute an alternative definition of points of local strict opposition. Using the geometric interpretation of the gradients, these points may also be called tangential points, i.e., points where level curves meet tangentially.

Definition 3: The parties are in local nonstrict opposition at a point $x \in \Re$ iff they are not in local strict opposition at $x$, i.e., iff it is possible for both parties to raise their utilities by moving an infinitesimal distance from $x$.

We also introduce two additional types of opposition based on the tension between the two parties at an offer in terms of the cooperativeness or conflict between the interests (utilities) of the two sides. Since each utility gradient represents the "central interests" or most preferred direction in which a party would like to move, if the two directions are close to each other, the two parties have more scope for cooperatively improving upon the compromise than if the two gradients were in largely opposing directions. ${ }^{3}$

Definition 4: The parties are in local weak opposition at a point $x \in \Re$ iff $\nabla U_{1}\left(x . \nabla U_{2}(x) \geq 0\right.$, i.e., iff the gradients at $x$ of the two utility functions form an acute or right angle.

Definition 5: The parties are in local strong opposition at a point $x \in \Re$ iff $\nabla U_{1}(x) \cdot \nabla U_{2}(x)<0$, i.e., iff the gradients at $x$ form an obtuse angle.

While local opposition allows us to discuss the nature of the opposition for an individual position-which is in or out of the feasible set $X$-such as an initial offer, a counter-offer, and a compromise, we can define global opposition which characterizes-and is restricted to-the entire set of feasible offers.

Definition 6: The parties are in global strict (nonstrict, weak, strong) opposition iff for every $x \in X$ they are in local strict (nonstrict, weak, strong) opposition.

Strict and nonstrict global oppositions are complementary cases. We will show that under global strict opposition the $C C$ is identical to the whole of $X$. In the case of global nonstrict opposition, the $C C$ lies on the frontier of $X$. There are also negotiation cases with local nonstrict opposition at some points of $X$ and strict opposition at others. In these cases the $(C C)$ is either on the frontier of $X$ or, more often, partially on the frontier and partially in the interior of $X$.

Another type of opposition that is intermediate in scale between local and global opposition is offer-pair opposition.

\footnotetext{
${ }^{3}$ This can be formally proved by considering the width of the band of directions that would result in joint improvement, given any two values for the gradients.
} 
It is defined, as the name implies, on a pair of points in offer space. Given that the two parties have made offers $x_{1}$ and $x_{2}$ respectively, the offer-pair opposition between the two parties is $\left.\nabla U_{1}(x) 1\right) \cdot \nabla U_{2}\left(x_{2}\right)$, and its nature can be characterized as strong or weak as before, based on whether its value is negative or positive. Offer-pair opposition is useful in modeling the relationship between the two parties at early stages of the negotiation when there is no single point that is genuinely under consideration as a final compromise. In such situations, the gradients at the offers indicate which directions are most preferred for each party, and they may choose to take this into account in concession making.

A curve that is closely allied to the $C C$ and useful in conceptualizing the preference and efficiency structure of the offer space is the following:

Definition 7: The tangential point curve $(T P C)$ is the set of points $x \in \Re$ at which the two utility functions satisfy $\nabla U_{1}(x)=-k \nabla U_{2}(x), k>0$, i.e., points at which the level curves of the two utility functions are tangential to each other.

Part of this curve coincides with the $C C$ under certain conditions (one of which is that the relevant portion of the TPC must be within the feasible set), and thus represents the efficient set under those conditions. However its more useful property is the fact that the gradients of both utilities, anywhere in $\Re$, always point roughly toward the TPC regardless of where the TPC may be located (in or out of the feasible set); this helps in visualizing the relationship between the two parties when depicted as a field of arrows. Put another way, "CC tends toward TPC," and a special case of this property is the result that when the TPC is entirely outside the feasible set, the Pareto-optimal set $(C C)$ must lie on the boundary of $X$, on those segments that are closest to the $T P C$. These aspects are explored in Section IV.

\section{OPPOSITION AND COMPROMISES}

\section{A. Conditions for Efficient Compromises}

The efficiency of the compromise $U M$ depends, for a given value of union's utility, on the management's utility function. Assume that $U M$ is efficient. The management's utility level curve or indifference curve, for simplicity assumed linear, is depicted in Fig. 4. Moving from $U M$ results in an increase in one party's utility at the expense of the other party's utility. This is because the parties are in strict opposition at $U M$.

Given a pair of analytic utility functions, the following theorem establishes purely local criteria that can be applied to determine whether a given position is an efficient point or not. Alternatively, the theorem can be viewed as the defining equation of the $C C$ (efficient set) in offer space. In unconstrained negotiations, only the first half of the theorem can apply, and the theorem effectively says that the TPC (tangential point curve) is identical to $C C$.

The results in this section can be easily generalized to higher dimensional vector spaces ( $m$ parties negotiating over $n$ issues), but we will keep the discussion to two dimensions to continue our case study and illustrate concepts geometrically. Further, these results hold under the slightly weaker requirement of quasiconvexity rather than convexity of $U_{U}(x), U_{M}(x)$, and $B(x)$.

Let $B(x)=0$ denote the equation of the boundary of $X$, defining $x \in X$ iff $B(x) \geq 0$. Then $\nabla B(x)$ is the outward normal direction to the boundary of $X$ at $x$.

Theorem 1: Offer $x^{*} \in X$ is efficient iff, either

A) $x^{*}$ is in the interior of $X$ and the local opposition at $x^{*}$ is strict, i.e.,

$$
\nabla U_{U}\left(x^{*}\right)=-k \nabla U_{M}\left(x^{*}\right)
$$

where $k$ is some positive constant, or

B) $x^{*}$ is on the boundary of $X$, and for some $\alpha, \beta \geq 0$

$$
\alpha \nabla U_{U}\left(x^{*}\right)+\beta \nabla U_{M}\left(x^{*}\right)=\nabla B\left(x^{*}\right)
$$

given convex/concave utility functions and a convex feasible set.

\section{Qualitative Explanation}

The theorem treats interior points and boundary points of the feasible set $X$ differently, and part A essentially says that an interior point is efficient iff it is a point at which some level curves of the two parties meet tangentially. A utility gradient indicates the direction in which its agent would most prefer to go if he only considered small immediate gains, and any direction making an acute angle with the gradient (i.e., any direction $d$ such that $\left.d \cdot \nabla U_{i}(x)>0\right)$ is a direction in which the utility can be improved. Therefore, if the two agents do not have exactly opposing gradients, there is a direction in which both can jointly improve their position from the current one, and the current position is not efficient. So local strict opposition is a necessary condition-and sufficient, with convex/concave utility functions-for an interior point to be efficient.

However, there may be no tangential points within $X$ (either because they are all outside $X$ or because there exist no tangential points at all for the given utility functions). In that case the efficient set will lie on the boundary of $X$. The local conditions that identify which boundary points are efficient are given in part B of the theorem, and essentially say that there is no direction for improvement within the set $X$ at a boundary efficient point. The expression $\alpha \nabla U_{U}\left(x^{*}\right)+$ $\beta \nabla U_{M}\left(x^{*}\right)$ defines a convex cone of directions at $x^{*}$, and when the outward normal to the boundary lies within this cone, any direction into $X$ at $x^{*}$ must make an obtuse angle with at least one of the two utility gradients, thereby failing to improve that utility.

Proof: A) Every point $x^{*}$ in $X$, must lie on some unique level curve of each utility function, and the level curve of $U_{U}(x)$ that passes through $x^{*}$ is described by the equation $U_{U}(x)=U_{U}\left(x^{*}\right)$. This level curve defines the boundary of a set $C=\left\{x_{X}\right.$ s.t. $\left.U_{U}(x) \geq U_{U}\left(x^{*}\right)\right\}$. Clearly, $x^{*}$ is efficient iff $U_{M}(x)$ is maximized within $C$ at $x^{*}$ (points outside $C$ are eliminated because those points are dominated with respect to the $U_{U}$ criterion, and points at which $U_{M}$ is not maximum are dominated by points at which it is). Thus we convert the problem of maximizing both $U_{U}(x)$ and $U_{M}(x)$ over $X$ to one of maximizing $U_{M}(x)$ given $U_{U}(x) \geq U_{U}\left(x^{*}\right)$. 
This constrained optimization problem is easily solved by the standard method of Lagrange (see, e.g., [27]), and immediately gives us the equation $\nabla U_{M}\left(x^{*}\right)+\lambda \nabla U_{U}\left(x^{*}\right)=$ $0, \lambda \geq 0$, as the general form of the solution, where $\lambda$ is the Lagrange multiplier. This equation is rewritten as (2) to convey the geometric properties of the solution.

B) Since the conversion of the two-criterion maximization problem to the constrained single-criterion maximization problem is general, we have the identical requirement, to maximize $U_{M}(\boldsymbol{x})$ given $U_{U}(\boldsymbol{x}) \geq U_{U}\left(\boldsymbol{x}^{*}\right)$ now, however, with an additional constraint, $B(x)=0$. Once again applying the method of Lagrange, we get the general form of the solution as $\nabla U_{M}\left(x^{*}\right)+\lambda \nabla U_{U}\left(x^{*}\right)+\mu \nabla B\left(x^{*}\right)=0, \lambda \geq 0, \mu \neq 0 . \mu$ is positive or negative according to whether the inner or outer normal direction of $B(x)$ is considered the gradient $\nabla B(x)$; in our case, with $B(x) \leq 0$ defining the region $X, \mu$ is negative. A simple rewriting of this expression obtains (3).

Note that parts $\mathrm{A}$ and $\mathrm{B}$ of the theorem are exhaustive but not mutually exclusive because when both conditions apply we have tangential points on the boundary.

We now discuss special cases of opposition and their implications for the nature of the $C C$.

\section{B. Strict Opposition}

Global opposition requires that at every point in $X$ the local opposition is always the same (strict or nonstrict).

Theorem 2: Assume that the agents' preferences differ. They are in global strict opposition in $X$ if $U_{U}$ and $U_{M}$ are linear and in local strict opposition at any one point in $X$.

Proof: If $U_{U}$ and $U_{M}$ are linear, the gradients of the two utilities are constant vectors all over $X$. Thus the two gradients align (in opposite directions) either everywhere or nowhere in $\mathrm{X}$. Therefore given any point of local strict opposition in $X$, global strict opposition is implied.

The converse is not true; given global strict opposition $\left(\forall x \in X \nabla U_{U}(x)=-k U_{M}(x)\right)$, the two utility functions need not be linear. Any pair of nonlinear functions such that one is the negative of the other satisfies this condition. A corollary to the theorem is that global strict opposition with one linear utility implies that the other utility function must also be linear.

The implication of Theorem 2 is that for the linear utilities and local strict opposition, the indifference curves are tangential at every point in $X$, and the whole set $X$ of feasible solutions is efficient. For linear utilities without local strict opposition, the entire interior of $X$ is inefficient and the efficient set lies on the boundary. (This is a special case of the theorem involving monotonicity below.) This is not the case for strictly convex (concave) utilities or when at least one of the utilities is nonlinear (Fig. 3, for example). Thus, in the case of strictly opposing parties, a linear approximation of the nonlinear utilities may result in significant errors and make impossible discrimination of offer efficiency.

From Theorem 2 it follows that in the linear case and strict initial opposition negotiations always terminate in an efficient compromise. We consider this special case, with condition (2) holding between the initial points, i.e., with $\nabla U_{U}\left(\boldsymbol{u}_{0}\right)=$

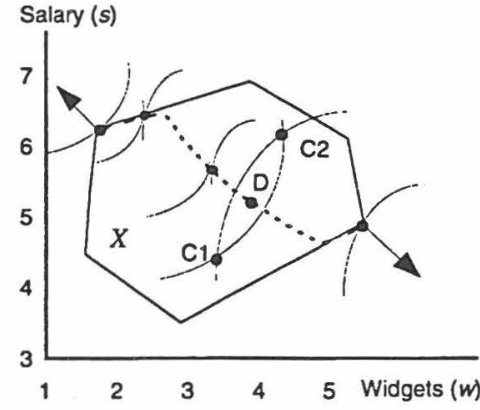

Fig. 5. Local strict opposition and inefficient compromises

$-k \nabla U_{M}\left(m_{0}\right)$. The nonlinear situation is illustrated in Fig. 5. The $C C$ is nonlinear and, depending on the initial offers and set $X$, it may initially be on the boundary of $X$. Strict local opposition (and thus efficiency) at the initial points does not ensure that negotiations will terminate in an efficient compromise, as the figure illustrates.

If $C 1$ or $C 2$ is the compromise then it is inefficient because the parties' utilities are not tangential at these points. Both parties may increase their respective utilities if they select any point from the dashed line. An efficient compromise is at point $D$.

\section{Nonstrict Opposition}

In the case of global nonstrict opposition, that is, if $\nexists x \in X$ such that $\nabla U_{U}(x)=-k \nabla U_{M}(x)$, the $C C$ lies on the boundary of $X$ because condition (a) of Theorem 1 does not apply in $X$ and thus (b) holds. We illustrate the case of global nonstrict opposition with a revised example of union-management negotiation and, as before, take the union's perspective. The two parties make the same initial proposals and counter-offers and reach the same compromise as previously. As we indicate in Fig. 6, the only difference is in the management's utility. The change in the utility from the one depicted in Fig. 4 is not very significant. In fact, it could be even smaller and the effect would be the same (for illustrative purposes we made a greater change in the indifference curves), that is the achieved compromise $U M$ would be inefficient. The same behavior of the two parties leads now to inefficiency because of different, than in Fig. 4, management's utility. In this case the $C C$ consists of two intervals $[U 0, P 2],[P 2, M 0]$.

The strength of opposition depends both on objectives and preferences. The management's objectives remain the same, it wants more of widgets and less of salary, but the trade-off coefficients change. The management is now more interested in salary (see Fig. 6) than it was in the example illustrated in Fig. 4. This small change moves negotiations from local strict opposition at some points within $X$ to global nonstrict opposition. This phenomenon has been observed in both reallife situations and in laboratory experiments [19]. Note that since this change may not be known to the other party, the sequence of offers and counter-offers may be the same as illustrated in Fig. 4. 


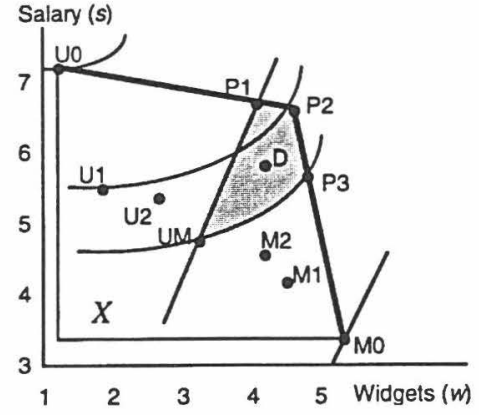

Fig. 6. Global nonstrict opposition and an inefficient compromise.

\section{Weak and Strong Opposition}

To consider the conditions for the weak and strong forms of opposition we make two assumptions. One, often used in economics and utility theory, is that the utilities are monotonic functions. The second assumption is that rational agents will not negotiate issues on which they are both indifferent. While strategic misrepresentation is possible, that is, a party may introduce an issue which has no implications for its own utility, this issue has to be at least relevant for the opponent. The implication of this assumption is that

$$
\begin{aligned}
& \forall x \in X: \partial U_{U}(x) / \partial x_{j} \\
& \quad=0 \rightarrow \partial U_{M}(x) / \partial x_{j} \neq 0, \quad j=1,2
\end{aligned}
$$

(and similarly with the utilities switched).

Theorem 3: Given $U_{U}(x)$ and $U_{M}(x)$ monotonic, and no irrelevant issues, if there is local weak opposition at any one point in $X$, then the two parties are in global nonstrict opposition over $X$.

It is not possible to prove a stronger result: e.g., local weak opposition does not imply global weak opposition; the strength of opposition can vary across $X$, even under the restrictive monotonicity assumption. However, the above theorem is quite useful since it concerns efficiency (global nonstrict opposition implies that efficient points are located only on the boundary, due to theorem 1).

Theorem 4 (complementary to 3 ). Given monotonic $U_{U}(x)$ and $U_{M}(x)$ and no irrelevant issues, if there is local strict opposition at any point within $X$, then the two parties are in global strong opposition.

Proofs of 3 and 4: Monotonicity implies that the derivatives do not change sign, i.e., each derivative is always nonnegative or nonpositive. Thus each of the components of the gradient vectors maintains the same sign throughout $X$. Since $\nabla U_{U}\left(x^{*}\right)=-k \nabla U_{M}\left(x^{*}\right)$ at an efficient point $x^{*}$, it follows that the corresponding components of the two gradient vectors must have opposite signs or be both zero at $x^{*}$. The latter possibility is ruled out by the assumption of no irrelevant issues. By monotonicity, the signs of the components at $x^{*}$ must hold throughout $X$. Consequently, if a tangential point exists within $X$ the dot product of the two vectors must be negative throughout $X$ (i.e., the opposition is globally strong).
Since local weak opposition at a point $x^{\prime} \in X$ is defined by

$$
\nabla U_{U}\left(x^{\prime}\right) \cdot \nabla U_{M}\left(x^{\prime}\right)>0
$$

it follows that it is not possible to have an efficient point within $X$ and simultaneously have weak opposition anywhere in $X$. That is, local weak opposition at any point in $X$ implies that there are no efficient points in the interior of $X$.

Theorem 5 (Generalization of Theorem 4). If $U_{U}(x)$ and $U_{M}(x)$ are monotonic and the gradients at any one point in $X$ are directed into opposite quadrants of $X$, then the two parties are in global strong opposition over $X$.

Proof: As explained with Theorem 3, monotonicity alone implies that each of the components of the gradient vectors maintains the same sign throughout $X$. When the two vectors are directed into opposite quadrants we have $\nabla^{T} U_{U}(x) \nabla U_{M}(x)<0$, which implies that each pair of corresponding components of the two vectors must be of opposite signs. Consequently the dot product of the two vectors must be negative in $X, \nabla U_{U}(x) \cdot \nabla U_{M}(x)<0$, and the opposition is globally strong.

A complementary theorem for gradients falling into the adjacent quadrants can be proved; this is a generalization of Theorem 3.

\section{RATIONALITIES AND EFFICIENCY}

In Section II, we discussed negotiations in which the two parties achieved a compromise with efficiency depending on the nature of the opposition. The efficiency conditions for different forms of opposition were developed in Section IV, and showed that any feasible offer might be an efficient solution, not just on the boundary of $X$. The conditions are specified for utility functions defined on the problem attributes and the parties' preferences, and the discussion of inefficient compromises thus far has been based on the structure of the underlying problem and preferences. In this section we will consider both problem-structural and behavioral reasons for inefficiency and present arguments as to why rational agents do not, and often should not, select an efficient compromise.

\section{A. Opposition and Efficiency}

The results obtained in Section IV-D also hold for offer-pair opposition. Therefore, knowledge of the opposition between the initial pair of offers provides information about the possible location of the efficient points. For example, given weak opposition between this pair, all the efficient points are on the boundaries of feasible set $X$. If the boundaries are known to both parties then "rational" agents can guess at and approximate the efficient set. If, however, the boundaries are not known or $X=\Re$, then rational, i.e., utility maximizing parties cannot achieve an efficient compromise. This is because given weak opposition any offer can be improved and there are no efficient compromises. Thus, feasibility plays a critical role in weakly opposing parties.

In the complementary theorem's situation, since the opposition is strong, efficient points are likely to be in the interior of $X$. This implies that the constraints defining $X$ may be insignificant and only the parties' utilities define efficient 
compromises. Thus, the acceptability of the offer to the parties becomes critical. The compromise's efficiency depends on accurate assessment of one's own party's and one's opponent's utilities.

Weak opposition allows for win-win negotiations while strong opposition suggests win-lose negotiations. A significant effort is often made to move the parties from the win-lose situation to win-win. This requires change in the opposition and, therefore, in their utilities. If successful, such a change requires joint agreement on the set of feasible solutions.

We assumed that utilities are stable over time. The problemstructural properties may, however, lead negotiators to change their utilities so that the set of available compromises is defined by objective constraints and not solely by subjective utilities. Attempts to modify utilities are usually considered desirable if they allow one to move from win-lose to win-win negotiations and expand the "pie." Thus, an initially efficient solution may become inefficient.

Weak (or strong or strict) opposition, when based on problem attributes, are structural notions directly characterizing the underlying interests of the parties. While they may be powerful in influencing the progress of negotiation and the outcomes, they are not the sole determining factors.

\section{B. Attributes of the Agent}

The attributes of the agent are all the characteristics that describe the decision maker and her behavior. These include knowledge and cognitive capability, risk attitude, ability and willingness to cooperate, sense of responsibility, power, and leadership. Preferences describe the values and tradeoffs of the agent but they are defined on the problem attribute values and do not take into account the agent's attributes.

Recognition of human cognitive limitations led Simon [29] to propose a much weaker form of rationality, namely bounded rationality. Obviously, negotiators who are only partially rational in the utilitarian sense and employ satisficing behavior may never be able to achieve efficient compromises. However, fully rational negotiators also have to balance the problem and the agent attributes.

The attributes of the agent may modify the choice mechanism. This has been illustrated with the consideration of the impact of relative power and time pressure on making offers in bargaining [30]. Perception of power may impact the scope of concessions but it may also cause selection of different offers for a given utility value. For a given concession level (measured with the utility function), the party may select an offer which yields the lowest utility of the opponent. This may lead to a different sequence of offers and an inefficient compromise. If such a compromise is achieved then rational negotiators should make a joint attempt to improve it.

In decision analysis, risk and risk attitude is associated with the outcomes and used in estimation of the expected utility. Post-settlement settlement introduces additional risk which negotiators may not be willing to accept. Opening the negotiation process anew may endanger what had already been achieved and, therefore, the negotiators may knowingly accept an inefficient compromise.

\section{Process Attributes}

Negotiation is a process that has its own process attributes such as time, effort, communication and monetary requirements. For example, in our simple case, the parties achieved a compromise based on the "split the difference" principle because they perceived that the differences between the two last offers (U2 and M2 in Fig. 4) do not warrant spending more time and effort on further discussions. This is an example of tradeoffs between the utility of a decision and the agent and process attribute values. Obviously, the split the difference approach may result in an inefficient compromise.

Consideration of the phases of negotiations introduce a qualitative difference between the initial offers, intermediary offers and the final offers [10]. The first offer is made only after the agent secured sufficient process resources in the prenegotiation phase. Offers made during the phases of differences exploration and their reduction deplete these resources. Thus, a potential offer is evaluated not only in its contribution to the final compromise but also in its resource implications for both parties [31].

Negotiations may be concluded if a party underestimated the resource requirements and its resources are depleted. More often, in the final bargaining phase the parties realize that the compromise is within reach. This may significantly change their perspective as the parties realize that the remaining resources can be used elsewhere. Since the process resources are often very expensive (e.g., the time of the executives and managers) and generic because they can be used elsewhere, there is strong competition for their distribution among different activities.

Negotiators with the achievement of a compromise become able to use their time and effort on other issues and solve other problems. As the union chief negotiator said, there is a constituency and many other issues have to be addressed. Students, in the experiments discussed in [6], completed the exercise and saw no reason to move from the achieved compromises. It may be that they thought that the time they saved in accepting the compromises would be better used elsewhere. The key issue here is that with the achievement of a compromise the trade-off analysis changes from the comparison of potential gains and losses between offers to comparison of these offers with expected gains from solving other problems using the available resources. Possible exceptions may occur with professional negotiators and advisors who are being paid for the process of securing the best possible compromise.

\section{Meta-Utility}

One may argue that the deficiency of the above discussion is its restriction to a utility defined only on the offer space, that is, on decision attributes. When all the attributes are taken into account then rational agents would always achieve efficient compromises. We accept this argument; on philosophical grounds the utilitarian approach will indeed result in efficiency. The question is how this can be translated into formal analysis and support mechanisms in the situation when some of the attributes are initially unknown and may never be known 
while other attributes are the result of the process which is to be controlled with the utility considerations. We do not know of any realistic approach which takes this into account. Game theory, negotiation analysis and other decision-theoretic approaches allow for only a few attributes of the agent and/or of the process and assume that their values are constant during negotiations.

An attempt to apply the utilitarian approach introduces a significant complexity to problem representation and solution. There are no grounds to assume that there is only one utility. On the contrary, Nozick [12] argues that at least three different utilities need be considered, the evidentially expected utility (a deterministic case is considered here), the causally expected utility and the symbolic utility. While efficiency can be determined with respect to one or two utilities, its absence cannot suggest that the decision maker is not rational. Due to the additional dimensions defining other attributes than those of the problem, the existence of competitive options and other decision problems, and different utilities one can assume that the rational agents will achieve an efficient compromise only in exceptional situations. Unless they disregard a number of aspects and features of the overall negotiation situation they may have important reasons not to follow the efficient frontier.

Even if all is known to all parties and the meta-utilities can be determined there is the issue of certainty. A negotiator may assume that the opponent is rational with probability of one but not with certainty. This allows the opponent to attempt to change this probability with the selection of inefficient offers. The argument may be such as the one given by the union negotiator in making offer $c 3$ (see Fig. 2). Varoufakis, [32, Ch. 6] shows that such a behavior may allow one party to achieve an improved compromise which, however, need not be efficient.

\section{IMPLICATION FOR ANALYSIS AND SUPPORT}

The prescriptive representations of the supported party assumes that all relevant problem, agent and process attributes are modeled and a meta-utility is constructed. There may be two reasons for such an effort. One is to better understand oneself and the problem, and such an effort is worthwhile. The other reason is to follow the model's prescriptions. We discussed the difficulty in constructing such a model but the most important drawback to following its prescriptions is the negotiator's subservience to mechanistic moves, inducing an inability to be creative and to understand the rationality guiding the opponent's moves.

We derived the concept of the opposition from that of utility and formulated theorems which allow negotiators to determine the set of efficient compromises. We also presented arguments against basing offer formulation on utility, and the limitations of utility-based approaches to negotiation analysis and support. This apparent contradiction can be resolved if-as we advocate here-opposition and its role are viewed in more general terms. This is because opposition may reflect some of the attributes of the agents and the process in addition to those of the problem.

We have introduced four types of opposition and their implications. These are clearly more basic and therefore of wider applicability than utilities; for any type of opposition we have many different utilities. We also think that opposition is a social phenomenon which can be easily understood because it is closely related to conflict or consonance.

Opposition can be approximated or modified with elements (attributes) that are difficult to measure and embedded in utility. The question which the negotiator has to answer is whether an additional issue or attribute weakens or strengthens the opposition and to what degree. The "degree" need not be precise as it is sufficient to know whether the two parties remain strictly opposed or whether the opposition moves from strong to weak or vice versa.

Specification of the nature of the opposition allows for approximation of the efficient set. In the case of weak opposition this set may be approximated with some accuracy because even without knowing the utility functions some boundaries will clearly be efficient. However, this seems less relevant than the knowledge of the critical boundaries. This knowledge allows the parties to focus their efforts on softening of the binding constraints. In the prenegotiation phase the parties assess the strength of the opposition. If it is weak then they identify the binding constraints and during the phase of difference exploration discuss the possibility of softening them. Then the parties may concentrate on offers which are defined by the agreed upon constraints or in their neighborhood.

A significant difference between the use of utility and opposition is that the latter opens up the possibility of including all perceived attributes (including agent and process attributes) without their formal representation and without a laborious process of preference elicitation. Utility is obtained through the bottom-up analytic approach that requires the decision maker to provide a comprehensive specification of all relevant attributes and to make a thorough and detailed comparison between them. Opposition is more of a qualitative concept and allows for a holistic approach. The decision maker may conduct analysis having an intuitive understanding of the strength of the opposition, with no specification of the underlying attributes and preferences.

Another difference is in the consideration of the process with respect to joint gains. Joint gains cannot be achieved if the parties are in strict opposition and do not wish to modify it. The efficiency of win/lose negotiations is defined by parties' utilities. Although this type of negotiation cannot be ruled out, experienced negotiators and mediators make an effort to change the nature of the opposition from strict to strong or even weak. Here too, the analysis of the opposition facilitates the process and may help to "increase the pie."

Weakening of the opposition moves the set of efficient compromises toward the boundaries of $X$. However, it seems less relevant whether the parties select an efficient compromise when they engage in the process of identifying differences and searching for similarities. Discussion on opposition and attempts to modify it allow for such a behavior. Obviously it involves changes of the utilities but this is the strength of the approach. Although it may be based on detailed analytical considerations, it allows broadening of one's perspective and calls for a creative use of resources. 
Application of analysis and support based on opposition in the example given in Section II does not necessarily disallow the construction and use of utility functions. However, these functions are no longer required to approximate the efficient solutions. The union, instead of trying to guess the management's utility may assess the type of opposition for the compromise solutions it contemplates.

We presented here an approach which has considerably weaker informational requirements than the approaches based on utility theory. Another important characteristic of our approach is that it is general and allows for consideration of different, dynamically changing and nonquantifiable attributes. Research on how these attributes can be incorporated and whether they should be introduced into opposition or treated separately is required.

One research direction to consider is the separation of forces pulling apart from these pulling together. Neither individual utilities nor opposition as discussed here allows to make a distinction between different efficient solutions. It is obvious, however, that only some of these solutions may be acceptable as compromises. Within utility framework different methods are proposed leading to aggregation of individual utilities. Practical use of these methods is minimal as they require the parties' discussion about their relative power and strength of conviction. Within the approach we propose, opposition may be complemented with cooperation which define the strength of the willingness to negotiate. Cooperation may allow to distinguish between efficient solutions, for example, in Fig. 2, cooperation for $U_{0}$ is weaker than for $e^{*}$. It also may allow to distinguish between utility-equivalent solutions $e 1, \cdots, e 6$. In that sense, the constructiveness of an offer mentioned by the union's negotiator (Section II-C) is related to the type (strength) of cooperation associated with the offer.

Another inviting research direction deals with generalization of the concept of opposition, or more generally, the "motivational force" of each party (defined by $\nabla U(\boldsymbol{x})$ when the utility $U(x)$ exists) to problems for which no utility function can possibly be defined. The existence of a utility function hinges upon the requirements that preferences be transitive, complete, continuous, and independent of the path by which a position is reached (equifinality). These assumptions have been rejected by many analysts as being problematic, e.g., people too often display circular preferences, and the satisfaction accrued by an agent depends on the history of the negotiation. In such cases, there provably does not exist a utility function defined solely on the offer space. However a "motivational force," corresponding to the gradient $\nabla U(x)$ does exist: recall that the gradient measured the preferred direction of local movement at a point, and the strength of the desire to move in that direction, and these notions still do apply meaningfully to the agents at that position. Therefore a measure of this "force" can be defined meaningfully in terms of the local preferences at a position. There is a close analogy with the mathematics of the electromagnetic field encountered in physics: motivation is like a force, and utility is like the potential corresponding to the force. The electric field is always the gradient of a potential ( $E=-\nabla V$, and the gain in $V$ in moving from one point to another is independent of the path) and one can do any analysis using the force field or the potential equivalently; this corresponds to the traditional utility assumptions. However, the magnetic field $H$ is in general not a gradient of a potential (curl $H \neq 0$ which implies nontransitivity and pathdependence); but formal analysis about a force is clearly meaningful even when a potential does not exist. This case corresponds to the way we have used the notion of opposition, focusing on the gradients more than on any utility function that a gradient may belong to. This analogy captures the spirit of our call for systematic methods for dealing with local preference information, without carrying the baggage of unrealistic rationality assumptions from utility theory.

\section{APPENDIX \\ DETERMINING THE REGION OF POSSIBLY EFFICIENT COMPROMISES}

In Fig. 1, the analyst has conservatively estimated the extreme values $p \in[0.3,3]$ of management's tradeoffs (slopes of the level curves of the utility function $\left.U_{M}(w, S)\right)$. Note that these extreme values do not depend on the point $e 3$ or any other specific point, but are estimated as bounds on $U_{M}$ 's slope for the entire region of interest. Using this information, and the fact that efficient points in the interior of $X$ must be tangential points (Theorem 1, part A) the set $E$ of possibly efficient compromises can be determined as follows. Computing the union's gradient from (1), we have

$$
\nabla U_{U}(w, s)=-\frac{1.951}{(w-5.596)^{2}} \boldsymbol{e}_{w}+0.5 \boldsymbol{e}_{s} .
$$

Consider first the extreme value of management's gradient $p=0.3$. Since $\vec{\nabla} U_{U}(w, S)=-k \vec{\nabla} U_{M}(w, S)$ for efficient points, we can find one extreme $C C$ (which is thus a boundary of $E$ ) by solving

$$
-\frac{1.951}{(w-5.596)^{2}} e_{w}+0.5 e_{s}=-k\left(0.3 e_{w}+e_{s}\right)
$$

which has as its solution $w=1.98$. Likewise, solving the same equation with $p=3$ results in $w=4.45$ as the other extreme $C C$. The region between $w=1.98$ and $w=4.45$ is thus the desired set $E$.

\section{ACKNOWLEDGMENT}

Comments and suggestions made by W. Michalowski and three referees are very much appreciated.

\section{REFERENCES}

[1] F. Alemi, P. Fos, and W. Lacorte, "A demonstration of methods for studying negotiations between physicians and health care managers," Decision Sci., vol. 21, pp. 633-641, 1990.

[2] L. Weingart, E. Hyder, and M. Pietrula, "Knowledge matters: The effect of tactical descriptions on negotiation behavior and outcome," Graduate School of Industrial Administration, Carnegie Mellon University, Pittsburgh, PA, 1995.

[3] M. Neale and G. Northcraft, "Experts, amateurs, and refrigerators: Comparing expert and amateur decision making in novel tasks," Organ. Perform. Hum. Decision Processes, vol. 38, pp. 305-317, 1986.

[4] D. Pruitt, Negotiation Behavior. New York: Academic, 1981.

[5] H. Raiffa, The Art and Science of Negotiation. Cambridge, MA: Harvard Univ. Press, 1982. 
[6] J. Teich, P. Korhonen, J. Phillips, and J. Wallenius, "Multiple issue negotiations: A study of BATNA's and corresponding inference surfaces," Helsinki School of Economics, Espoo, Finland, unpublished manuscript, 1995 .

[7] E. F. McClennen, Rationality and Dynamic Choice. Foundational Explorations. Cambridge, U.K.: Cambridge Univ. Press, 1990.

[8] J. K. Sebenius, "Negotiation analysis: A characterization and review," Manage. Sci., vol. 38, no. 1, pp. 18-38, 1992.

[9] M. A. Bazerman and M. A. Neale, "Negotiator rationality and negotiator cognition: The interactive roles of prescriptive and descriptive research," in Negotiation Analysis, H. P. Young, Ed. Ann Arbor, MI: Univ. of Michigan Press, 1991, pp. 109-130.

[10] P. H. Gulliver, Disputes and Negotiations. New York: Academic, 1979.

[11] J. G. March, "Bounded rationality, ambiguity, and the engineering of choice" in Decision Making: Descriptive, Normative, and Prescriptive Perspectives, D. E. Bell, H. Raiffa, and A. Tversky, Eds. Cambridge, U.K.: Cambridge Univ. Press, 1988, pp. 33-57.

[12] R. Nozick, The Nature of Rationality. Princeton, NJ: Princeton Univ. Press, 1993.

[13] R. A. Shweder, "Divergent rationalities," in Methatheory in Social Science, D. W. Fiske and R. A. Shweder, Eds. Chicago, IL: Univ. of Chicago Press, 1986, pp. 163-196.

[14] D. A. Lax and J. Sebenius, The Manager As Negotiator. New York: Free Press, 1986.

[15] R. Fisher, E. Kopelman, and A. K. Schneider, Beyond Machiavelli: Tools for Coping with Conflict. Cambridge, MA: Harvard Univ. Press, 1994.

[16] R. Fisher and W. Ury, Getting to "Yes"-Negotiating Agreement Without Giving In. New York: Penguin, 1983.

[17] A. Rangaswamy and G. R. Shell, "Using computers to realize joint gains in negotiations: Toward an electronic bargaining table," in Computer Assisted Negotiation Mediation Symp., Harvard Law School, Cambridge MA, May 26-27, 1994.

[18] P. Young, Ed., Negotiation Analysis. Ann Arbor, MI: Univ. of Michigan Press, 1991.

[19] T. Darling and J. L. Mumpower, "Modeling cognitive influences on the dynamics of negotiation," Emerging Technologies and Applications, Proc. HICSS. Los Alamitos, CA: IEEE Computer Society Press, 1990, pp. 22-30.

[20] R. D. Luce and H. Raiffa, Games and Decisions. New York: Wiley. 1957.

[21] O. R. Young, "Strategic interaction and bargaining" in Bargaining. Formal Theories of Negotiations, O. R. Young, Ed. Urbana, IL: Univ. of Illinois Press, 1975.

[22] K. Chatterjee, "Incentive compatibility in bargaining under uncertainty," Q. J. Econ., pp. 717-726, 1982.

[23] P. C. Cramton, "Bargaining with incomplete information: An infinitehorizon model with two-sided uncertainty," Rev. Econ. Stud., pp. 579-593, 1984

[24] D. Fudenberg and J. Tirole, "Sequential bargaining with incomplete information," Rev. Econ. Stud., pp. 221-247, 1983.

[25] M. Verkama, "Distributed methods and processes in games of incomplete information," Ph.D. dissertation, System Analysis Lab., Helsinki Univ. of Technol., Espoo, Finland, 1994.
[26] A. J. Jones, Game Theory: Mathematical Models of Conflict. Chichester, U.K.: Ellis Horwood, 1980, pp. 210-217.

[27] _ Game Theory: Mathematical Models of Conflict. New York: Wiley, 1980.

[28] P. Y. Papalambros and D. J. Wilde, Principles of Optimal Design. Cambridge, U.K.: Cambridge Univ. Press, 1988.

[29] H. A. Simon, The New Science of Management Decision. New York Harper and Row, 1960

[30] P. Balakrishnan and J. Eliashberg, "An analytical process model of two-party negotiations," Manage. Sci., vol. 41, no. 2, pp. 226-243, 1995

[31] A. Rapoport, I. Erev, and R. Zwick. "An experimental study of buyerseller negotiation with one-sided incomplete information and time discounting," Manage Sci., vol. 41, no. 3, pp. 377-394, 1995.

[32] Y. Varoufakis, Rational Conflict. Oxford, U.K.: Blackwell, 1991

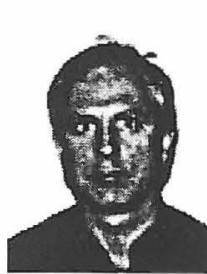

Gregory E. Kersten received the Ph.D. degree from the Warsaw School of Economics, Warsaw, Poland, in 1981 .

He joined the Carleton University School of Business, Ottawa, Ont., Canada, in 1984, where he is currently Professor of Decision Sciences and Information Systems, and the Director of the Centre for Computer Assisted Management. In 1997. he joined the International Institute for Applied Systems Sciences, Laxenburg. Austria, as a Senior Researcher. He has authored and coauthored more than 60 articles in refereed journals and conference proceedings, and 16 book chapters. He is the Principal Investigator of InterNeg, a World Wide Webbased negotiation project.

Dr. Kersten is vice-chairperson of the INFORMS Group Decision and Negotiation College, Departmental Editor of the Group Decision and Negotiation Journal, and a member of several editorial boards.

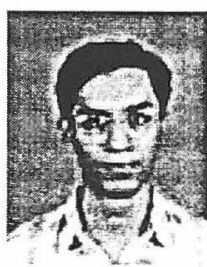

Sunil J. Noronha (S'88-M'91) received the B.Tech degree in electrical engineering from the Indian Institute of Technology, Madras, India, in 1987 and the Ph.D. degree from the Computer Science and Automation Department, Indian Institute of Science. Bangalore, in 1993.

In 1994, he joined School of Business, Carleton University, Ottawa, Ont., Canada, as a Postdoctoral Fellow and worked on the Negoplan and InterNeg projects in negotiation support. Since 1997, he has been a Research Staff Member at the IBM T. J. Watson Research Center, Yorktown Heights, NY. His research interests include topics in artificial intelligence, decision support systems, data mining, and electronic commerce.

Dr. Noronha is a member of the ACM and INFORMS. 



\section{ORDERING INFORMATION}

Orders must include the publication number and should be sent to the Publications Department, International Institute for Applied Systems Analysis, A-2361 Laxenburg, Austria.

Telephone: +432236807

Telefax: +43223671313

E-mail: molina@iiasa.ac.at

A full list of IIASA publications is available at http://www.iiasa.ac.at 
International Institute for

Applied Systems Analysis

A-2361 Laxenburg, Austria 